\title{
Evaluation method of access capacity based on TSC in distribution network
}

\author{
Fengchuan $\mathrm{Nie}^{1, \mathrm{a}}$, Minfang Peng ${ }^{1}$, Yong Xu${ }^{1}$, Haiyan Zhang ${ }^{1}$, Liang $\mathrm{Zhu}^{2}$, Hongwei Che ${ }^{2}$, Zhengyi Liu ${ }^{2}$ \\ ${ }^{1}$ College of Electrical and Information Engineering, Hunan University, Changsha, 410082, China \\ ${ }^{2}$ State Grid Hunan Electric Power Company, Changsha 410007, China \\ aemail: 1151952048@qq.com
}

\begin{abstract}
In order to select suitable lines and reduce approval process effectively, the article presents a method to evaluate access capacity based on total supply capability. Firstly, after making a brief introduction to total supply capability, we define access capacity and give two access capacity indexes, which are substation supply access capacity and network transfer access capacity. Secondly, based on constrained information of substation supply capability、network transfer capability、 node voltage and node current, we present a model to evaluate access capacity and calculate it. At last, based on this method the article evaluate access capacity of a distribution network line with 44 nodes, the results show that this method can reflect the access capacity in distribution network line accurately.
\end{abstract}

Keywords-Distribution System; Access Capacity Evaluation; Total Supply Capability.

\section{INTRODUCTION}

A study on access capacity of the distribution network can select suitable lines and reduce approval process effectively.

For the distribution network, there are a lot of researches on the distribution planning, but there is no evaluation method of access capacity. It proposed the residual power supply capacity, the analysis and train of thought are given in [1]. but there is no concrete model and constrained condition to evaluate. In [2] when calculating TSC, it put forward the concept of available supply capacity, but each main transformer and the link of the available supply capacity are not covered. So the research on access capacity of line and transformer in distribution network is little.

\section{DEVELOPMENT OF CALCULATING TSC}

The calculation of TSC experienced three stages[2-8]: the first stage was evaluating power supply capacity based on the distribution capacity, such as the method of capacity-load ratio; the second stage was based on power restoration, such as maximum load multiples method; the third stage was based on N-1 security criteria, substation capacity and power restoration. Two indexes extended from TSC expressed as the following:

SSC: The TSC of a distribution system when each feeder is not linked with any other.
NTC: The amount of TSC increased by interconnections among feeders.

\section{LAYER-BY-LAYER BACK/FORWARD SWEEP DISTRIBUTION LOAD FLOW ALGORITHM}

The algorithm calculate node voltage and node current consists of the following two steps:

Push forward the power: according to the Hierarchy matrix of network, push forward the power from the last layer to layer 1, update Branch terminal power step by step, then get the lower branch for every branch from Hierarchy matrix.

Return voltage: according to the Hierarchy matrix of network, return the power from layer 1 to the last layer, update Branch terminal node voltage step by step. that is, update node voltage of the end in the next layer.

\section{DEFINITION OF ACCESS CAPACITY IN THE DISTRIBUTION NETWORK}

On the basis of the existing transmission contract,the available transmission capacity (ATC) [9] is the remanent actual physical transmission network. According to ATC we define access capacity in distribution system which is the maximum power, and takes into the $\mathrm{N}-1$ safety criterion and the actual constraints account in a certain power supply area. And two indexes extended from access capacity expressed as the following:

Substation Supply Access Capacity: the access capacity of a distribution system when each feeder is not linked with any other. That is, any substation transformer has no connection with other substation transformers.

Network Transfer access capacity: the amount of access capacity increased by interconnections among feeders.

V. THE MODEL TO EVAlUATE ACCESS CAPACITY BASED ON TSC

A. Links of the distribution network

1) Link of the rated capacity of main transformer [2]

$$
X_{i}+R_{i} T_{i} \leq k R_{i}
$$


Where $R_{i}=$ rated capacity of transformer $\mathrm{i} ; X_{i}=$ access capacity of transformer $\mathrm{i} ; T_{i}=$ loading rate of transformer $\mathrm{i}$; $k$ =overload factor of transformer for a short time, assuming all transformers have the same $k$ value $(\mathrm{k}=1.2)$.

2) Link of substation supply capability

$$
\sum_{i \in Z_{i}} X_{i}+\sum_{i \in Z_{i}} R_{i} T_{i} \leq S S C_{i}
$$

Where $S S C_{i}=S S C$ of main transformer $\mathrm{i} ; Z_{i}=$ substation $\mathrm{i} ; i \in Z$ represents that the main transformer of substation $\mathrm{i}$.

3) Link of rated capacity of output transmission line

$$
Y_{i j} \leq C_{i j}
$$

Where $Y_{i j}=$ the access capacity of the line which connect transformer $\mathrm{i}$ and transformer $\mathrm{j}$; $C_{i j}=$ capacity limitation of the line wh $\mathrm{h}_{\mathrm{i}} \mathrm{ch}$ connect transformer $\mathrm{i}$ and transformer $\mathrm{j}$.

4) Link of network transfer capability

$$
\sum Y_{i j} \leq N T C
$$

Where NTC=the amount of TSC increased by interconnections among feeders.

5) Link of node voltage and node current [10-12]

$$
\begin{aligned}
& V_{v}^{\min } \leq V_{v} \leq V_{v}^{\max } \\
& I_{a_{v}-v} \leq I_{a_{v}-v}{ }^{\max }
\end{aligned}
$$

Where $a_{v}$ is the father node of $v ; V_{v}^{\min }, V_{v}, V_{v}^{\max }$ are voltage magnitudes lower limit ,actual voltage and voltage magnitudes; $I_{a_{v}-v}, I_{a_{v}-v}{ }^{\max }$ are actual current and current magnitudes through branch $a_{v}-v, v \in S_{i}, i=1,2, \ldots, k$.

B. Linear programming model

$$
\begin{aligned}
& \max \sum_{i \in Z} X_{i}+\sum_{i \in Z} Y_{i j} \\
& \text { s.t. }\left\{\begin{array}{l}
X_{i}+R_{i} T_{i} \leq k R_{i} \\
\sum_{i \in Z_{i}} X_{i} \leq S S C_{i} \\
Y_{i j} \leq C_{i j} \\
\sum Y_{i j} \leq N T C \\
V_{v}^{\min } \leq V_{v} \leq V_{v}^{\max }, \quad v \in S_{i}, i=1,2, \ldots, k \\
I_{a_{v}-v} \leq I_{a_{v}-v}^{\max }, \quad v \in S_{i}, i=1,2, \ldots, k
\end{array}\right.
\end{aligned}
$$

(8)

In (7), we take the maximum sum of substation supply access capacity and network transfer access capacity as the objective. The (8) is based on constrained information of substation supply capability, network transfer capability, node voltage and node current. In the article, we assume that the node voltage and the node current meet the condition.

\section{ANALYSIS OF EXAMPLE}

We use a distribution network line [2] with 44 nodes as an example shown in Fig1.

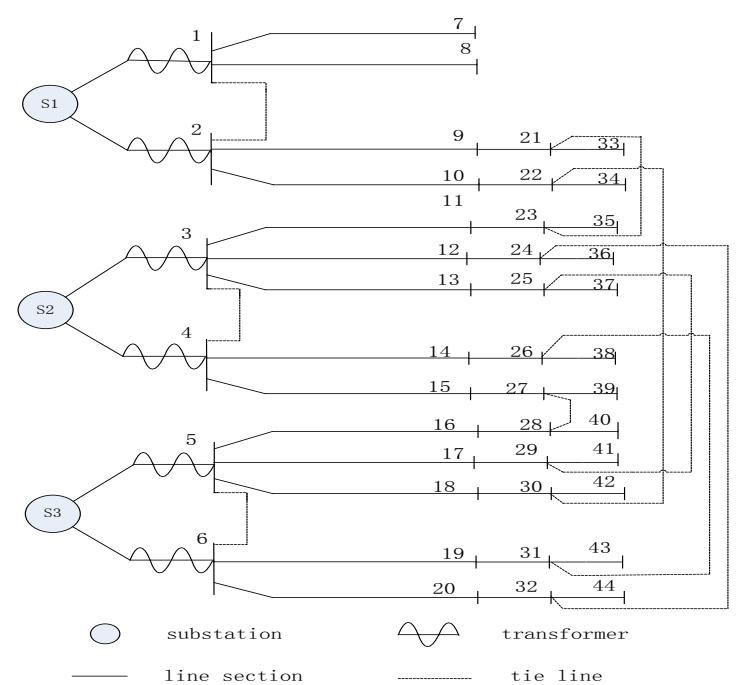

Fig.1. the test distribution network line with 44 nodes

In the figure above, 1-44 represent nodes, S1-S3 represent three transformer substation, broken line represent output transmission line and solid line represent distribution line.

\section{A. Data of the distribution network}

The following is date of transformer substation and line. All the main feeders are JKLYJ-185.

Table 1. output transmission line date of the test distribution network

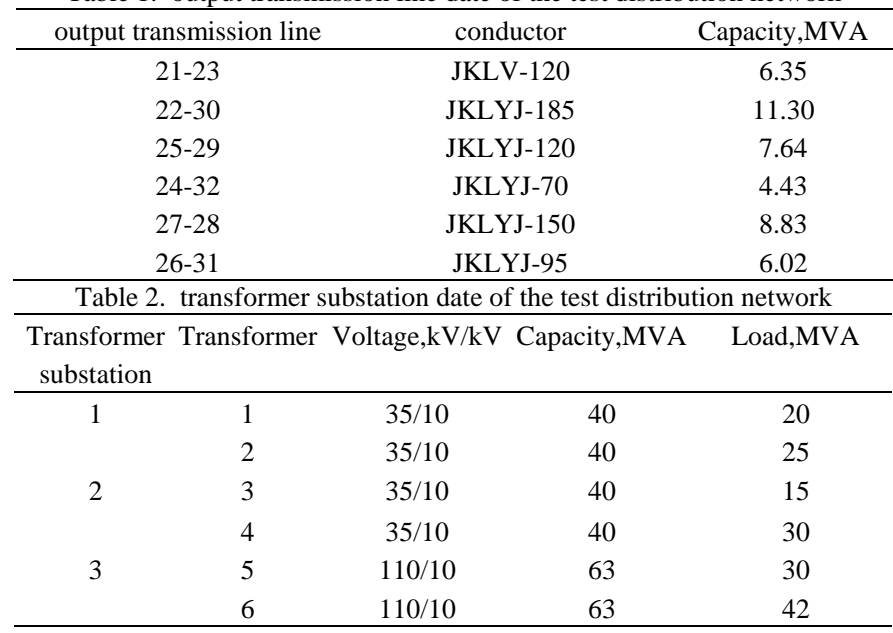

B. Substation supply access capacity and network transfer access capacity

The total access capacity is $176.5 \mathrm{MVA}$, Substation supply access capacity is $132.0 \mathrm{MVA}$, network transfer access capacity is $44.6 \mathrm{MVA}$. 
Table 3. result of the test distribution network

\begin{tabular}{cccc}
\hline Transforme] & $\begin{array}{c}\text { Substation supply } \\
\text { access capacity }\end{array}$ & $\begin{array}{c}\text { Output } \\
\text { transmission } \\
\text { line }\end{array}$ & $\begin{array}{c}\text { Network transfer } \\
\text { access } \\
\text { capacity }\end{array}$ \\
\hline 1 & 22.0 & $21-23$ & 6.4 \\
2 & 22.0 & $22-30$ & 11.3 \\
3 & 14.6 & $25-29$ & 7.6 \\
4 & 14.6 & $24-32$ & 4.4 \\
5 & 29.4 & $27-28$ & 8.8 \\
6 & 29.4 & $26-31$ & 6.0 \\
\hline
\end{tabular}

\section{CONCLUSION}

Reference to the definition of the available transmission capacity (ATC), we define access capacity and give two access capacity indexes, which is substation supply access capacity and network transfer access capacity. Then based on TSC and taking constraint information of substation supply capability, network transfer capabilit, node voltage and node current into account; we present a model taking the maximum sum of substation supply access capacity and network transfer access capacity as the objective. On the basis of the above, we present the calculation linear programming model and use lingo to calculate. At last, it proves effective and feasible.

Along with the advancement of marketing-distributiondispatching work, the demand for the efficiency of the power supply enterprise service is higher and higher. So the further research can focus on how to form the power supply scheme quickly based on access capacity, shorten the waiting time, even zero waiting.

\section{ACKNOWLEDGMENT}

This work is supported by National Natural Science Foundation of China under Grant No.61173108 and 61472128, Hunan Provincial Natural Science Foundation of China No.10JJ2045 as well as 14JJ2150 and State Grid Corporation Science and technology projects of China No.5216A514001K.

\section{References}

[1] Liu Lifeng, Liu yong, Ma Jiming. Research and Realization for surplus power supply capacity analysis system in distribution network[J].Shanxi Electric Power, 2011,39 (5):44-46.

[2] Xiao Jun,Gu Wenzhuo,Guo Xiaodan,Wang Chengshan,LI Fangxing. Power supply capability model for distribution system[J]. Automation of Electric Power Systems, 2011,35 (24):47-52.

[3] Gu Wenzhuo. Definition, model and calculation method of total supply capability of distribution system [D]. Tianjin: Tianjin University, 2012.

[4] XIAO Jun,Li Fangxing,Gu Wenzhuo,et al.Total supply capability and its extended indices for distribution system: definition, model, calculation and applications[J].IET Generation, Transmission \& Distribution,2011,5(8):869-876.

[5] Xiao Jun, Guo Xiaodan, Wang Chengshan, Zu Guoqiang. The nature of the maximum distribution network power supply capacity model solution[J]. Automation of Electric Power Systems,2013,37(16):59-65.

[6] Xiao Jun, Gong Xiaoxu, Wang Chengshan. Comparative validation between the maximum supply capacity of the distribution and $\mathrm{N}-1$ security Calibration[J]. Automation of Electric Power Systems, 2012,36(18): 86-91.

[7] $\mathrm{Yu} \mathrm{Xu}$, Chen Zhiming. Evaluation of power supply capability in medium voltage distribution networks based on fuzzy theory [C].Guilin:Machine Learning and Cybernetics,2011:10-13.

[8] Jun Han,Ge Shaoyun,Liu Hong,Liu Yang,Zhang Xin. Structural optimization of urban distribution network for improving power supply capability[C]. Shanghai : Electricity Distribution (CICED),2012:10-14.

[9] Li Guoqing, Li Song, Li Xiaojing. Available Transfer Capability Calculation Considering FACTS Controllers[J].Proceedings of the CSEE, 2009,29 (19) : 36-42.

[10] Yan Wei, Liu Fang, Wang Guanjie, Xu Guoyu, Huang Shanglian. Layer-by-layer back/forward sweep method for radial distribution load flow[J].Proceedings of the CSEE, 2003,23 (8) : 76-80.

[11] JU Yuntao, Wu Wenchuan, Zhang Boming, Sun Hongbin. Convergence Problem of Forward/Backward Sweep Power Flow Caused by the Internal Impedance of Distributed Generators [J].Proceedings of the CSEE, 2014,34 (34):6185-6189.

[12] Zhang Qin, Zhou Buxiang, Lin Nan, Zhang Jianmei. Improved power flow algorithm for distribution networks based on Zbus algorithm and forward/backward sweep method [J]. Proceedings of the Chinese Society of Universities for Electric Power System and its Automation, 2012,24(6):73-77. 